\title{
Correction to: The Effectiveness of Two Methods of Prescribing Load on Maximal Strength Development: A Systematic Review
}

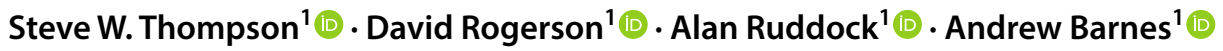

Published online: 2 January 2020

(c) The Author(s) 2019

\section{Correction to: Sports Medicine}

https://doi.org/10.1007/s40279-019-01241-3

While typesetting the entries of the Table 1 were incorrectly aligned. The correct Table 1 has been copied below.

The original article has been corrected.

The original article can be found online at https://doi.org/10.1007/ s40279-019-01241-3.

Steve W. Thompson

s.w.thompson@shu.ac.uk

1 Academy for Sport and Physical Activity, Sheffield Hallam University, Sheffield, UK 
Table 1 Study characteristics

\begin{tabular}{|c|c|c|c|c|c|c|c|c|}
\hline Study & $\begin{array}{l}\text { Participants } \\
(n)\end{array}$ & $\begin{array}{l}\text { Groups } \\
(+ \text { participant } \\
\text { numbers }(n))\end{array}$ & $\operatorname{Sex}(n)$ & $\begin{array}{l}\text { Age } \\
\text { (years } \pm \mathrm{SD})\end{array}$ & $\begin{array}{l}\text { Body mass } \\
(\mathrm{kg} \pm \mathrm{SD})\end{array}$ & $\begin{array}{l}\text { Stature } \\
(\mathrm{cm} \pm \mathrm{SD})\end{array}$ & $\begin{array}{l}\text { Resistance train- } \\
\text { ing experience }\end{array}$ & $\begin{array}{l}\text { Participant char- } \\
\text { acterisation }\end{array}$ \\
\hline $\begin{array}{l}\text { Weiss et al. } \\
\text { (1988) [33] }\end{array}$ & 56 & $\begin{array}{l}\text { RT (28) } \\
\text { C (28) }\end{array}$ & $\begin{array}{l}\text { M (28) } \\
\text { F (28) }\end{array}$ & $20.8 \pm 1.8$ & NR & NR & NRT $<3$ months & Healthy \\
\hline $\begin{array}{l}\text { Braith et al. } \\
\quad \text { (1993) [34] }\end{array}$ & 58 & $\begin{array}{l}\text { RT (47) } \\
\text { C (11) }\end{array}$ & $\begin{array}{l}\text { M (33) } \\
\text { F (25) }\end{array}$ & $\begin{array}{l}24.0 \pm 4.0 \\
25.0 \pm 5.0\end{array}$ & $\begin{array}{l}70.1 \pm 9.0 \\
74.3 \pm 14.5\end{array}$ & $\begin{array}{l}174.0 \pm 6.3 \\
172.6 \pm 6.6\end{array}$ & $\mathrm{NRT}<1$ year & Untrained \\
\hline $\begin{array}{l}\text { Moss et al. } \\
\text { (1997) [35] }\end{array}$ & 31 & $\begin{array}{l}\text { RT-G90 (9) } \\
\text { RT-G35 (11) } \\
\text { RT-G15 (10) }\end{array}$ & M & $\begin{array}{l}22.7 \pm 3.4 \\
24.0 \pm 3.4 \\
22.9 \pm 2.8\end{array}$ & $\begin{array}{l}75.8 \pm 5.6 \\
83.2 \pm 8.8 \\
78.1 \pm 10.4\end{array}$ & $\begin{array}{l}179.0 \pm 6.8 \\
185.7 \pm 8.5 \\
182.6 \pm 6.7\end{array}$ & Well-trained & $\begin{array}{l}\text { University } \\
\text { students (non- } \\
\text { dominant } \\
\text { arm = control } \\
\text { group) }\end{array}$ \\
\hline $\begin{array}{l}\text { Bell et al. } \\
\quad(2000) \text { [36] }\end{array}$ & 21 & $\begin{array}{l}\mathrm{RT}(11) \\
\mathrm{C}(10)\end{array}$ & $\begin{array}{l}\text { M (12) } \\
F(9)\end{array}$ & $22.3 \pm 3.3$ & $73.4 \pm 11.6$ & $176.0 \pm 9.3$ & NRT & $\begin{array}{l}\text { University } \\
\text { students }\end{array}$ \\
\hline $\begin{array}{l}\text { Campos et al. } \\
\text { (2002) [37] }\end{array}$ & 31 & $\begin{array}{l}\mathrm{RT}-\mathrm{LR}(9) \\
\mathrm{RT}-\mathrm{IR}(7) \\
\mathrm{RT}-\mathrm{HR}(7) \\
\mathrm{C}(5)\end{array}$ & M & $\begin{array}{l}21.1 \pm 1.5 \\
20.7 \pm 2.9 \\
20.4 \pm 3.5 \\
31.6 \pm 9.8\end{array}$ & $\begin{array}{l}80.1 \pm 8.4 \\
79.5 \pm 7.8 \\
70.2 \pm 9.5 \\
80.8 \pm 23.3\end{array}$ & $\begin{array}{l}179.8 \pm 6.5 \\
179.6 \pm 7.4 \\
174.3 \pm 8.6 \\
178.1 \pm 5.5\end{array}$ & NRT $<6$ months & Healthy \\
\hline $\begin{array}{l}\text { McBride et al. } \\
\text { (2003) [38] }\end{array}$ & 28 & $\begin{array}{l}\mathrm{RT}-\mathrm{S} 1(9) \\
\mathrm{RT}-\mathrm{M} 6(9) \\
\mathrm{C}(10)\end{array}$ & $\begin{array}{l}\mathrm{F}(13) \\
\mathrm{M}(15)\end{array}$ & $\begin{array}{l}22.1 \pm 3.4 \\
20.0 \pm 1.22 \\
22.4 \pm 1.89\end{array}$ & $\begin{array}{l}83.7 \pm 29.4 \\
70.7 \pm 23.0 \\
70.6 \pm 7.8\end{array}$ & $\begin{array}{l}172.8 \pm 10.5 \\
169.4 \pm 11.8 \\
171.3 \pm 7.2\end{array}$ & NRT ( $<6$ months) & Untrained \\
\hline $\begin{array}{l}\text { Willoughby } \\
\text { (2004) [39] }\end{array}$ & 22 & $\begin{array}{l}\text { RT (12) } \\
\text { C (10) }\end{array}$ & M & $20.9 \pm 2.76$ & $78.7 \pm 6.2$ & $176.5 \pm 7.1$ & NRT $<6$ months & Untrained \\
\hline $\begin{array}{l}\text { Tricoli et al. } \\
\text { (2005) [40] }\end{array}$ & 14 & $\begin{array}{l}\text { RT (7) } \\
\text { C (7) }\end{array}$ & M & $22.0 \pm 1.5$ & $73.4 \pm 10.4$ & $179.4 \pm 8.8$ & $\begin{array}{c}\text { NRT }<3 \text { months } \\
\quad \text { (trained prior) }\end{array}$ & College students \\
\hline $\begin{array}{l}\text { Rana et al. } \\
\text { (2008) [41] }\end{array}$ & 16 & $\begin{array}{l}\text { RT (9) } \\
\text { C (7) }\end{array}$ & $\mathrm{F}$ & $\begin{array}{l}20.6 \pm 1.9 \\
22.9 \pm 2.4\end{array}$ & $\begin{array}{l}64.1 \pm 7.9 \\
72.5 \pm 15.0\end{array}$ & $\begin{array}{l}165.6 \pm 4.9 \\
163.6 \pm 4.5\end{array}$ & NRT & Untrained \\
\hline $\begin{array}{l}\text { Tanimoto } \\
\text { et al. (2008) } \\
\text { [42] }\end{array}$ & 24 & $\begin{array}{l}\text { RT (12) } \\
\text { C (12) }\end{array}$ & M & $\begin{array}{l}19.5 \pm 0.5 \\
19.8 \pm 0.7\end{array}$ & $\begin{array}{l}63.8 \pm 4.0 \\
64.2 \pm 4.0\end{array}$ & $\begin{array}{l}174.8 \pm 4.3 \\
174.3 \pm 7.2\end{array}$ & NRT & Healthy \\
\hline $\begin{array}{l}\text { Terzis et al. } \\
(2008) \text { [43] }\end{array}$ & 17 & $\begin{array}{l}\text { RT (11) } \\
\text { C (6) }\end{array}$ & M & $22.0 \pm 1.0$ & $85.0 \pm 4.0$ & $184.0 \pm 3.0$ & NRT $<1$ year & P.E students \\
\hline $\begin{array}{l}\text { Hartmann } \\
\text { et al. (2009) } \\
\text { [44] }\end{array}$ & 40 & $\begin{array}{l}\mathrm{RT}-\mathrm{SPP}(13) \\
\mathrm{RT}-\mathrm{UP}(14) \\
\mathrm{C}(13)\end{array}$ & M & $\begin{array}{l}24.31 \pm 3.2 \\
25.14 \pm 4.0 \\
24.77 \pm 3.1\end{array}$ & $\begin{array}{l}84.7 \pm 11.2 \\
79.4 \pm 10.4 \\
74.4 \pm 12.1\end{array}$ & $\begin{array}{l}183.9 \pm 7.2 \\
177.6 \pm 7.5 \\
180.5 \pm 8.1\end{array}$ & $\begin{array}{l}\text { RT in BP (mini- } \\
\text { mum } 1 \mathrm{RM} \text { of } \\
100 \mathrm{~kg})\end{array}$ & $\begin{array}{l}\text { Sport science } \\
\text { students }\end{array}$ \\
\hline $\begin{array}{l}\text { Cormie et al. } \\
\text { (2010) [45] }\end{array}$ & 16 & $\begin{array}{l}\mathrm{RT}(8) \\
\mathrm{C}(8)\end{array}$ & M & $23.9 \pm 4.8$ & $79.8 \pm 12.0$ & $180.0 \pm 6.4$ & $\begin{array}{l}\text { NRT (Technically } \\
\text { proficient in BS) }\end{array}$ & Healthy \\
\hline $\begin{array}{l}\text { Chtourou } \\
\text { et al. (2012) } \\
\text { [46] }\end{array}$ & 30 & $\begin{array}{l}\text { RT-MTG } \\
(10) \\
\text { RT-ETG } \\
(10) \\
\text { C (10) }\end{array}$ & M & $22.9 \pm 1.3$ & $72.0 \pm 8.8$ & $180.0 \pm 5.0$ & NRT $<6$ months & P.E students \\
\hline $\begin{array}{l}\text { Weier et al. } \\
\text { (2012) [47] }\end{array}$ & 12 & $\begin{array}{l}\mathrm{RT}(6) \\
\mathrm{C}(6)\end{array}$ & $\begin{array}{l}M(6) \\
F(6)\end{array}$ & $\begin{array}{l}20 \pm 0.8 \\
22 \pm 0.6\end{array}$ & NR & NR & NR & $\begin{array}{l}\text { University } \\
\text { students }\end{array}$ \\
\hline $\begin{array}{l}\text { Naclerio et al. } \\
\text { (2013) [48] }\end{array}$ & 32 & $\begin{array}{l}\mathrm{RT}-\mathrm{LV}(6) \\
\mathrm{RT}-\mathrm{MV}(6) \\
\mathrm{RT}-\mathrm{HV}(8) \\
\mathrm{C}(7)\end{array}$ & $\begin{array}{l}\mathrm{M}(20) \\
\mathrm{F}(12)\end{array}$ & $\begin{array}{l}23.3 \pm 1.2 \\
23.3 \pm 1.4 \\
23.9 \pm 2.0 \\
22.1 \pm 1.1\end{array}$ & $\begin{array}{l}66.4 \pm 11.0 \\
71.4 \pm 8.5 \\
69.4 \pm 12.5 \\
71.1 \pm 14.2\end{array}$ & $\begin{array}{l}169.9 \pm 8.4 \\
173.3 \pm 7.6 \\
173.0 \pm 9.8 \\
169.7 \pm 6.9\end{array}$ & $\mathrm{NRT}<5$ years & $\begin{array}{l}\text { Team sports } \\
\text { athletes } \\
\text { Soccer }(20)(\mathrm{M}) \\
\text { Volleyball (12) } \\
\text { (F) }\end{array}$ \\
\hline $\begin{array}{l}\text { Aguiar et al. } \\
\text { (2015) [49] }\end{array}$ & 18 & $\begin{array}{l}\text { RT (9) } \\
\text { C (9) }\end{array}$ & M & $\begin{array}{l}20.9 \pm 2.0 \\
20.0 \pm 1.8\end{array}$ & $\begin{array}{l}73.7 \pm 9.4 \\
75.0 \pm 8.8\end{array}$ & $\begin{array}{l}173.8 \pm 6.9 \\
176.4 \pm 8.1\end{array}$ & NRT $<6$ months & Healthy \\
\hline $\begin{array}{l}\text { Akagi et al. } \\
\text { (2016) [50] }\end{array}$ & 23 & $\begin{array}{l}\text { RT (13) } \\
\text { C (10) }\end{array}$ & M & $22.1 \pm 1.1$ & $61.4 \pm 5.8$ & $170.6 \pm 5.8$ & $\begin{array}{l}\text { NRT upper body } \\
\text { (<6 months })\end{array}$ & Healthy \\
\hline
\end{tabular}


Table 1 (continued)

\begin{tabular}{|c|c|c|c|c|c|c|c|c|}
\hline Study & $\begin{array}{l}\text { Participants } \\
(n)\end{array}$ & $\begin{array}{l}\text { Groups } \\
(+ \text { participant } \\
\text { numbers }(n))\end{array}$ & $\operatorname{Sex}(n)$ & $\begin{array}{l}\text { Age } \\
(\text { years } \pm S D)\end{array}$ & $\begin{array}{l}\text { Body mass } \\
(\mathrm{kg} \pm \mathrm{SD})\end{array}$ & $\begin{array}{l}\text { Stature } \\
(\mathrm{cm} \pm \mathrm{SD})\end{array}$ & $\begin{array}{l}\text { Resistance train- } \\
\text { ing experience }\end{array}$ & $\begin{array}{l}\text { Participant char- } \\
\text { acterisation }\end{array}$ \\
\hline \multirow{3}{*}{$\begin{array}{l}\text { Botton et al. } \\
\text { (2016) [51] }\end{array}$} & \multirow[t]{3}{*}{43} & RT-UG (14) & \multirow[t]{3}{*}{ F } & $24.8 \pm 1.4$ & $60.8 \pm 6.4$ & $163.0 \pm 6.5$ & \multirow[t]{3}{*}{ NRT $<3$ months } & \multirow[t]{3}{*}{ Healthy } \\
\hline & & $\mathrm{RT}-\mathrm{BG}(15)$ & & $24.3 \pm 3.7$ & $57.0 \pm 4.8$ & $160.2 \pm 5.8$ & & \\
\hline & & C (14) & & $22.7 \pm 2.8$ & $58.0 \pm 5.7$ & $163.6 \pm 6.2$ & & \\
\hline \multirow{3}{*}{$\begin{array}{l}\text { Wirth et al. } \\
\text { (2016) [52] }\end{array}$} & \multirow[t]{3}{*}{120} & $\mathrm{RT}-\mathrm{SQ}(43)$ & \multirow[t]{3}{*}{ M } & $23.7 \pm 2.7$ & $81.6 \pm 9.8$ & $181.7 \pm 7.5$ & \multirow[t]{3}{*}{ NR } & \multirow[t]{3}{*}{ Students } \\
\hline & & RT-LP (40) & & $23.8 \pm 2.3$ & $80.5 \pm 8.1$ & $180.1 \pm 7.0$ & & \\
\hline & & C (37) & & $25.1 \pm 2.1$ & $78.2 \pm 8.5$ & $181.0 \pm 5.7$ & & \\
\hline \multirow{2}{*}{$\begin{array}{l}\text { Jarvis et al. } \\
\text { (2017) [53] }\end{array}$} & \multirow[t]{2}{*}{21} & RT (11) & \multirow{2}{*}{$\begin{array}{l}M(15) \\
F(6)\end{array}$} & $27.5 \pm 3.2$ & $72.7 \pm 18.0$ & $169.6 \pm 10.3$ & \multirow[t]{2}{*}{$\mathrm{RT}>1$ year } & \multirow{2}{*}{$\begin{array}{l}\text { Collegiate } \\
\text { athletes }\end{array}$} \\
\hline & & $\mathrm{C}(10)$ & & $27.2 \pm 3.4$ & $76.4 \pm 11.5$ & $176.2 \pm 7.9$ & & \\
\hline \multirow{4}{*}{$\begin{array}{l}\text { Souza et al. } \\
\text { (2018) [54] }\end{array}$} & \multirow[t]{4}{*}{33} & $\mathrm{RT}-\mathrm{NP}(8)$ & \multirow[t]{4}{*}{ M } & $25.6 \pm 6.3$ & $79.5 \pm 13.0$ & $172.8 \pm 6.1$ & \multirow[t]{4}{*}{ NRT (<6 months) } & \multirow[t]{4}{*}{ College students } \\
\hline & & $\mathrm{RT}-\mathrm{TP}(9)$ & & $25.0 \pm 7.0$ & $76.0 \pm 9.9$ & $175.3 \pm 5.7$ & & \\
\hline & & RT-UP (8) & & $24.4 \pm 5.2$ & $74.9 \pm 4.2$ & $176.8 \pm 5.3$ & & \\
\hline & & C (8) & & $25.1 \pm 3.3$ & $76.8 \pm 11.7$ & $173.6 \pm 6.8$ & & \\
\hline
\end{tabular}

Mean $\pm S D$ standard deviation

IRM 1 repetition maximum, $B G$ bilateral training group, $C$ control, $c m$ centimetres, $B P$ bench press, $B S$ back squat, $E T G$ evening training group, $F$ female, $G 1515 \%$ load group, G35 35\% load group, G90 90\% load group, $H R$ high-repetition group, $H V$ high volume, $I R$ intermediate-repetition group, $k g$ kilograms, $L P$ leg press group, $L R$ low-repetition group, $L V$ low volume, $M$ male, $M 6$ six set training group, $M T G$ morning training group, $M V$ moderate volume, $n$ number, $N P$ non-periodised group, $N R T$ no resistance training, $N R$ not reported, $P . E$ Physical Education, $R T$ resistance training, $S 11$ set training group, $S P P$ strength-power periodisation, $S Q$ squat group, $T P$ traditional periodisation group, $U G$ unilateral training group, $U P$ daily undulating periodised group

Open Access This article is licensed under a Creative Commons Attribution 4.0 International License, which permits use, sharing, adaptation, distribution and reproduction in any medium or format, as long as you give appropriate credit to the original author(s) and the source, provide a link to the Creative Commons licence, and indicate if changes were made. The images or other third party material in this article are included in the article's Creative Commons licence, unless indicated otherwise in a credit line to the material. If material is not included in the article's Creative Commons licence and your intended use is not permitted by statutory regulation or exceeds the permitted use, you will need to obtain permission directly from the copyright holder. To view a copy of this licence, visit http://creativecommons.org/licenses/by/4.0/. 